\title{
Retention of Knowledge and Clinical Skills by Medical Students: A Pro- spective, Longitudinal, One-Year Study Using Basic Pediatric Cardiology as a Model
}

\author{
Fernando Amaral ${ }^{1}$ and Luiz Ernesto de Almeida Troncon ${ }^{2, *}$ \\ ${ }^{I}$ Pediatric Cardiologist and Adult Congenital Heart Cardiologist, Former faculty member of the University of Ribeirao \\ Preto (UNAERP) Brazil \\ ${ }^{2}$ Ribeirao Preto Medical School, University of Sao Paulo, Brazil
}

\begin{abstract}
Background: Retention of early-taught knowledge and fundamental clinical skills, albeit essential in medical education, has not been extensively investigated. This study is aimed at assessing prospectively the retention of knowledge and skills related to communication with patients and the physical examination by fourth year medical students after an introductory course in basic pediatric cardiology.

Methods: Three cohorts of fourth year medical students aged 21-26 years volunteered for the study. All students $(\mathrm{N}=$ 130) were assessed immediately after the teaching period and after six months $(\mathrm{N}=42)$ and after one year $(\mathrm{N}=21)$ later. Assessments included a 40 multiple-choice question (MCQ) test and an 8-station objective structured clinical examination (OSCE) focused on communication with patients, physical examination and interpretation of diagnostic tests (chest radiographs and electrocardiograms). Cases were portrayed by either real, rehearsed patients or standardized patients. Student performance was assessed by trained staff members using structured checklists.

Results: For all aspects of knowledge assessed, scores obtained in the second and the third assessment were significantly lower than those verified in the first assessment. There were no significant differences between the three assessments regarding overall clinical performance and data for communication with patients and physical examination. Concerning diagnostic tests interpretation, scores obtained in the second and third assessment were significantly lower than those verified in the first assessment. Paired analysis of the results obtained for the 21 students who took the three assessments showed similar results, except for a trend for improvement in clinical skills.

Conclusions: Medical student retention of clinical sciences knowledge follows a pattern similar to that found in other scientific domains, characterized by progressive decay after initial acquisition. In contrast, fundamental clinical skills, such as communication with patients and physical examination are substantially retained, which could be ascribed to continuous practice. These findings should be taken into account for devising instructional strategies for enhancing student knowledge and clinical skills maintenance.
\end{abstract}

Keywords: Medical students; Educational assessment; Performance appraisal; Clinical skills; Medical Education.

\section{BACKGROUND}

Individual proficiency in medical practice is highly, albeit not solely, dependent on knowledge and skills continuously acquired since the undergraduate period and during further training stages. Retention of previously taught subjects has been a long-standing concern for medical educators [1]. It is generally assumed that a large proportion of what is taught for medical students is lost in subsequent years [1,2]. Data from a recent review (1) indicate that basic biomedical sciences knowledge taught earlier in medical school is forgotten in a pattern similar to that of knowledge in other scientific domains. Available evidence indicates that the non

\footnotetext{
*Address correspondence to this author at the Department of Medicine Hospital das Clínicas - Campus da USP, Ribeirao Preto, State of Sao Paulo, Brazil, Zip Code (CEP): 14048-900; Tel: +55 (16) 3602 2457;

Fax: +55-16-3602 0226; E-mail: ledatron@fmrp.usp.br
}

use or non practice of previously taught knowledge and skills seems to be the main determinant of the degree of loss [1, 35]. Nevertheless, studies on retention of taught subjects is still scarce and there is hardly any investigation concerning knowledge related to clinical sciences.

The factors determining retention and decay of motor skills have also been a matter of concern to psychophysiologists and educators and the results of some studies indicate that there is substantial skill loss with nonuse $[6,7]$. The rise of simulation-based education focusing on the acquisition of specific skills relevant to medical practice has renewed the interest in both knowledge retention and specific strategies aiming at the maintenance of what is learned. A number of studies have shown that concentrated learning and training, as well as repeated testing are able to improve performance related to specific skills, such as those involved in advanced cardiac life support $[8,9]$, advanced trauma life 
support [10], general surgery [11] and laparoscopic skills [12], drug administration skills [13], cardiopulmonary resuscitation skills [14], central venous catheter insertion skills [15], critical obstetrical events [16] and general knowledge on life support [17], amongst others.

As far as fundamental clinical skills are concerned, there is data showing that interviewing and history-taking skills also decline over the following years after initial teaching, thus showing a pattern similar to those found for basic sciences knowledge [18-20]. Nevertheless, the retention of physical examinations skills has only been investigated in very specific settings, such as eye examinations [21] and studies on more general skills are even more scarce.

Since retention of fundamental clinical skills comprises an important matter in any medical specialty, and both the monitoring of maintenance and the detection of skill decay during the medical school period would be crucial for the student, as it would allow for intervention before clinical practice starts investigations focusing on both clinical knowledge and skills retention are pertinent. The purpose of this study was therefore to assess how medical students retain the knowledge and the fundamental clinical skills intensively taught during a short period of time.

\section{METHODS}

\section{Settings}

This study was carried out at the University of Ribeirao Preto (UNAERP) undergraduate medical program. By the time this investigation was carried out, this private medical school received 50 students per semester, who were admitted shortly after finishing high school for a six-year course. The local curriculum was mainly traditional, teacher-centred, comprising four semesters of integrated basic sciences, one semester of pre-clinical disciplines and three semesters of clinical disciplines. Nevertheless, both in pre-clinical and clinical disciplines, student activities were carried out mainly in outpatient clinics, where opportunities for practicing clinical skills were frequent. The two final years were spent in internships in major clinical areas, such as Internal Medicine, General Surgery, Pediatrics and Gynecology \& Obstetrics.

\section{Study Design}

This was a prospective study focusing on retention of knowledge and clinical skills by fourth-year medical students after a short teaching period in basic pediatric cardiology. For one year, cohorts of students were followed up and assessed periodically using a standard set of multiple-choice questions (MCQ) test and an objective structured clinical examination (OSCE), which were applied in a random order within an one-week interval. The MCQ items were written following standard guidelines [22]. The OSCE was designed and carried out following the original description [23], with appropriate adaptations to the local context [24]. The standardized patients who staffed some of the stations were trained according to Barrows' recommendations [25].

\section{Subjects}

Three cohorts of fourth year medical students aged between 21-26 years volunteered for the study. All students (N
$=130,68$ female) were assessed immediately after the teaching period, but the number of students attending the assessment decreased progressively when the assessment was reapplied six months later $(\mathrm{N}=42)$ and one year $(\mathrm{N}=21)$ later. There were no significant differences between the subgroup of 21 students who took all the three assessments and the remaining 109 students comprising the first cohort concerning age, gender and school achievement, as measured by ordinary grades in regular disciplines.

\section{The Teaching Period}

After finishing an introductory course on basic clinical skills (history-taking, physical examination and clinical reasoning), students attended a two-week intensive module on cardiovascular diseases, comprising of specific lectures, topics and cases discussions and practical activities with patients in hospital wards and outpatients clinics, which were carried out for the whole day ( 80 hours). This module included specific sessions (20 hours) focusing on pediatric cardiology comprising three formal lectures, two sessions of clinical topics discussion and various clinical activities with previously selected patients, who presented specific symptoms and signs and were invited to attend the sessions. All the teaching sessions on pediatric cardiology were conducted by the same faculty member. After this module, students attended regular clinical teaching sessions and lectures focusing on other areas of Medicine for one semester before starting the internship, but there were no specific activities concerning pediatric cardiology after the initial teaching sessions.

\section{Assessment}

The MCQ test comprised 40 items, which were randomly divided into knowledge about the basis of clinical skills (17 items) and knowledge about diseases and pathophysiology mechanisms (23 items). For each item there were five answers with only one correct answer. Test contents were closely related to what was taught in the teaching sessions and all items were the same for the three assessments.

The OSCE comprised eight stations, each one including a variable set of individual tasks. There were two history taking stations (a breathless infant and a school boy with chest pain), one communication station (breaking good news: diagnostic report to a mother of a child with an innocent murmur), three cardiovascular physical examination stations (one innocent and two pathological murmurs), and two diagnostic test interpretation stations (electrocardiogram and chest radiographs). Cases in the history-taking, communication and physical examination stations were portrayed by standardized patients, including both real, rehearsed patients and simulated patients. Stations lasted eight minutes each and the student performance was assessed by a trained staff member inside the room, who used structured checklists containing from 12 up to 17 items. All the students had been exposed to the stations tasks and clinical scenarios during the teaching sessions.

For the analysis of the results, the history taking and communication skills stations were grouped together (communication), as were the three physical examination stations and the two exams interpretation stations. 
Results from both the MCQ test and the OSCE were normalized so as to be expressed in a range from zero to ten. For each student, the overall result of the OSCE was expressed as the mean of the scores obtained in each station.

As far as content validity was concerned, the blueprint and the printed material of both the MCQ test and the OSCE cases were previously appraised by four independent external referees from other institutions [26]. All referees reported that in general there was an adequate matching between questions, clinical tasks and cases to the course instructional objectives, and that both MCQ items and instructions for the OSCE stations and the checklist items descriptors were clear and pertinent. Items that were criticized at this point were carefully reviewed and edited, so as to comply with referee's opinions. One of the referees, who has experience in structured examinations of clinical skills with simulated patients, also monitored case portrayal by standardized patients in the OSCE and found it to be adequate. Additionally, after every examination, students were asked to answer by way of a structured questionnaire focusing on aspects of exam organization, clarity of instructions, pertinence and degree of complexity of the cases included and more than $90 \%$ of respondents $(\mathrm{n}=126)$ rated these aspects as "good" or "very good".

With regard to reliability, results for both the MCQ test and the OSCE were split in two halves (even-numbered and odd-numbered questions and stations) and no statistically significant differences between split scores were found [27].

\section{Data Analysis}

Results from the three assessments (immediately after, six months and one year after the teaching sessions) were compared using non- parametric analysis of variance (KruskalWallis test). Data concerning the performance of the 21 students submitted to the three consecutive assessments were specifically analyzed using paired analysis of variance (Friedman test). Differences were regarded as significant for $P$ value equal or less than 0.05 . A tendency for significance was considered when the $\mathrm{P}$ value was between 0.05 and 0.10 .

\section{Ethical Considerations}

This work was part of a quality improvement project in medical education aimed specifically at introducing OSCE for undergraduate students in one institution using the exper- tise acquired in another; and accordingly did not require formal ethics approval. Nevertheless, the study was conducted in accordance with the Declaration of Helsinki and the national ethical guidelines. Moreover, the whole study project was approved by the UNAERP Educational Board and informed consent was obtained from all participating students, after providing them with detailed information about the nature and purpose of the investigation. Participation in the study was completely voluntary, and all participants were free to leave the study at any time without any repercussions. All individual results remained anonymous during the study and were analyzed confidentially. The use of standardized patients (simulated and real, rehearsed patients) for teaching and assessment was previously approved by an institutional Ethics Committee (Statement HCRP Number 724/95).

\section{RESULTS}

\section{Knowledge Retention}

Results concerning knowledge retention obtained in the three assessments are shown in Table 1. For all aspects of knowledge assessed, scores obtained in the second and the third assessment were significantly lower than those verified in the first assessment, thus indicating that a significant decay in knowledge retention was verified. A similar finding was observed in the paired analysis of results from the 21 students who took the three assessments, as shown in Fig. (1). There were no significant differences between this subgroup and the overall group of students concerning the results obtained in the first assessment.

\section{Clinical Skills Retention}

Results concerning clinical skills retention are shown in Table 2. There were no significant differences between the three assessments regarding overall clinical performance and data for communication and physical examination. Regarding diagnostic tests interpretation, scores obtained in the second and third assessment were significantly lower than those verified in the first assessment, thus indicating a significant decay in retention of this skill.

Paired analysis of data from the 21 students who took the three assessments (Fig. 2) showed that scores for overall clinical performance and for communication skills obtained

Table 1. Results on Three Different Assessments (A1, A2, A3) Carried Out with a Standard MCQ Test for Various Aspects of Knowledge Related to Pediatric Cardiology. A2 and A3 were Carried Out Six Months and One Year after A1, Respectively. Results are Expressed as Median and (Range). N: Number of Students Assessed

\begin{tabular}{|c|c|c|c|}
\hline Knowledge Aspects & $\begin{array}{c}\mathrm{A1} \\
(\mathrm{N}=\mathbf{1 3 0})\end{array}$ & $\begin{array}{c}A 2 \\
(\mathrm{~N}=42)\end{array}$ & $\begin{array}{c}\text { A3 } \\
(\mathrm{N}=21)\end{array}$ \\
\hline Overall & $\begin{array}{c}6.7 \\
(4.0-9.2)\end{array}$ & $\begin{array}{c}6.0 * \\
(4.2-7.7)\end{array}$ & $\begin{array}{c}5,7 * * \\
(4.0-7.7)\end{array}$ \\
\hline Clinical skills-related & $\begin{array}{c}7.6 \\
(4.1-9.4)\end{array}$ & $\begin{array}{c}7.0^{*} \\
(4.7-9.4)\end{array}$ & $\begin{array}{c}6,5^{* *} \\
(5.3-8.2)\end{array}$ \\
\hline
\end{tabular}

* - $\mathrm{p}<0.01$ versus $\mathrm{A} 1$; ** - $\mathrm{p}<0.0001$ versus $\mathrm{A} 1$. 


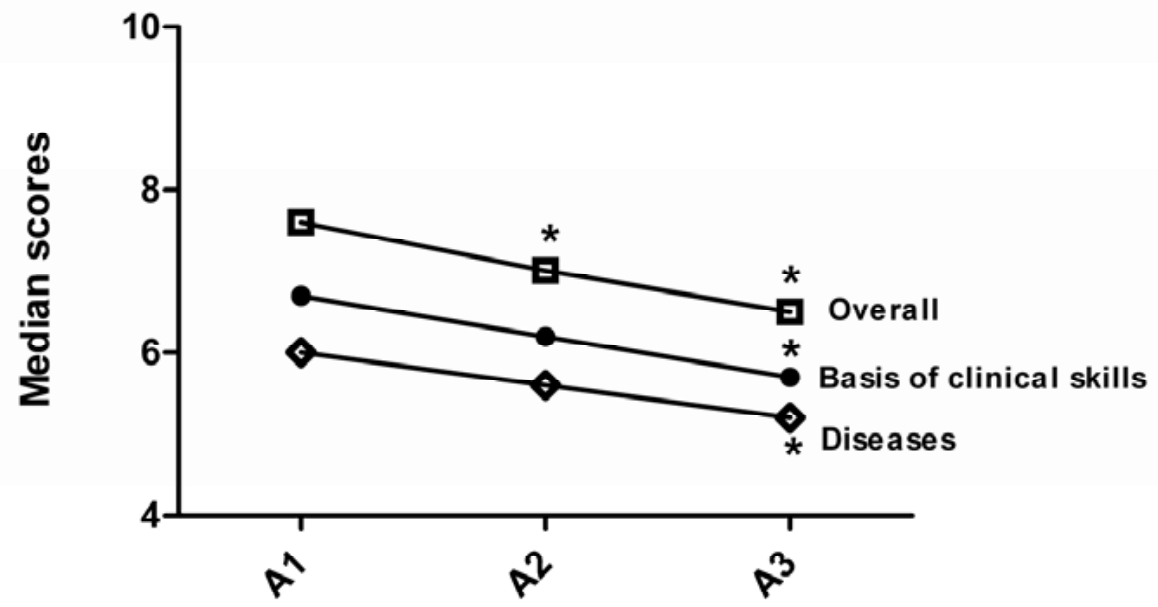

Fig. (1). Paired scores (medians) obtained by 21 students who took the three assessments (A1, A2, A3) carried out with a standard multiple choice questions (MCQ) test for various aspects of knowledge related to pediatric cardiology. A2 and A3 were carried out six months and one year after A1, respectively. * - p< 0.05 versus A1.

Table 2. Results on Three Different Assessments (A1, A2, A3) Carried Out with an Objective Structured Clinical Examination (OSCE) for Various Aspects of Clinical Skills Performance Using Pediatric Cardiology Cases. A2 and A3 were Carried Out Six Months and One Year After A1, Respectively. Results are Expressed as Median and (Range). N: Number of Students Assessed

\begin{tabular}{|c|c|c|c|}
\hline Clinical Skills Aspects & $\begin{array}{c}\mathbf{A 1} \\
(\mathbf{N = 1 3 0})\end{array}$ & $\begin{array}{c}\mathbf{A 2} \\
(\mathbf{N}=\mathbf{4 2})\end{array}$ & $\begin{array}{c}\mathbf{A 3} \\
(\mathbf{N}=\mathbf{2 1})\end{array}$ \\
\hline \hline Overall & 7.9 & $(2.5-10.0)$ & 7.9 \\
$(2.0-10.0)$ & 7.0 & $(3.0-10.0)$ \\
\hline Communication & $(4.29 .0)$ & 7.3 & $(6.0-9.8)$ \\
\hline Physical examination & 9.3 & $(7.3-10.0)$ & 9.2 \\
\hline Exams interpretation (electrocardiogram and chest radiographies) & $(4.0-9.5)$ & $6.5 *$ & $(7.2-10.0)$ \\
\hline
\end{tabular}

* - $\mathrm{p}<0.01$ versus $\mathrm{A} 1$.

in both the second and the third assessments were higher than in the first one $(\mathrm{p}=0.07)$, thus indicating a trend for improvement. No significant differences were found regarding physical examination. As far as the results for diagnostic interpretation tests were concerned, scores obtained in the third assessment were significantly lower than those verified in the first assessment, thus indicating again a significant decay in retention of this specific clinical skill. There were also no significant differences between this subgroup and the overall group of students concerning the results obtained in the first assessment.

\section{DISCUSSION}

The results of our study indicates that in the two semesters subsequent to an intensively taught introductory course of basic pediatric cardiology there was a significant decay of knowledge retention, whereas performance regarding clinical skills remained stable and even tended to improve, except for the interpretation of diagnostic tests, such as chest radiographs and the electrocardiogram, which also decayed significantly.

Although more than $80 \%$ of students dropped out from the study for unknown reasons, the performances of the remaining 21 volunteers on both the MCQ and the OSCE applied in the first assessment were similar to the overall group, which allowed us to regard them as a representative sample. The reasons for dropping out of the study were not investigated, but could putatively be ascribed to loss of interest, overload of other learning activities, or both.

Since the items included in the MCQ test were the same in the three assessments, we cannot rule out that a memorization effect had occurred from one assessment to another, which would imply an even higher loss of knowledge throughout the three semesters of our study in case the items had been changed. Also, repeated testing has been regarded 


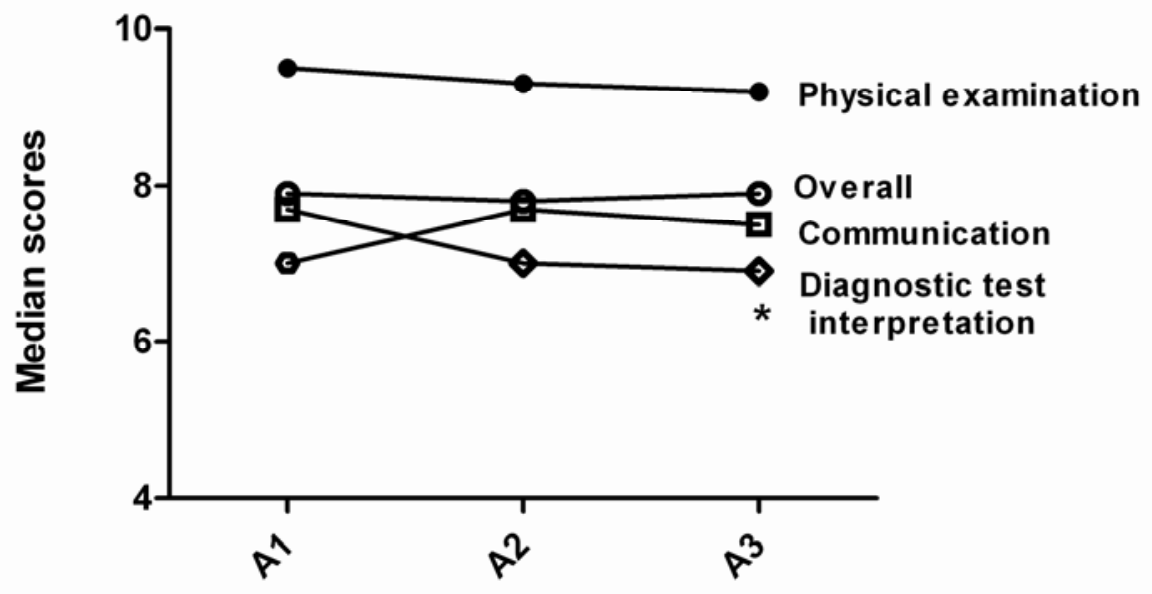

Fig. (2). Paired scores (medians) obtained by 21 students who took the three assessments (A1, A2, A3) carried out with an objective structured clinical examination (OSCE) for various aspects of clinical skills performance related to pediatric cardiology. A2 and A3 were carried out six months and one year after A1, respectively. * - p< 0.05 versus A1.

as a measure that could prevent knowledge decay [28], which may lead one to assume that an even higher loss would be found if students were tested only once, one year after the teaching period. As far as the clinical skills assessment is concerned, the pediatric cardiology model adopted seems to us particularly adequate for an OSCE, since the physical findings of the most frequent congenital heart defects varies little from patient to patient [29] and therefore different patients could be used, in order to minimize such memorization effect.

The most likely cause for the decay in knowledge retention observed in this study is lack of use of previously taught knowledge [1, 3, 4]. Accordingly, contents related to pediatric cardiology deals with very specific aspects, so that the probability of student exposure to this subject after the initial teaching period and training was thought to be small. Also, the "priority competition" phenomenon [6] might also have a role, due to the considerable amount of information provided to the students in their learning activities in the periods between assessments, which has long been assumed to occur in medical schools with a traditional curriculum [30]. It is interesting to note, however, that the knowledge applied at the patient's bedside has been reported to have a slower decay rate [31], which could account for the relatively better performance on the clinical skills-related knowledge questions (Table 1) in our results when compared to the knowledge about disease items. Nevertheless, the magnitude of loss was relatively small, as the difference between the medians for the scores for the first (6.7) and the last (5.7) overall knowledge assessment (Table 1) was near to $15 \%$. This is in line with the findings of a recent investigation that showed that the loss of knowledge related to basic biomedical sciences by medical students is relatively small and even doctors who had graduated many years previously show substantial retention of knowledge taught in medical school [4]. Therefore, whether the degree of loss observed in the current study is educationally relevant or not remains to be further investigated.

The maintenance, with a tendency for improvement, of student performance regarding communication skills is in contrast to previous findings showing that after an intensive course designed to develop effective communication and interviewing skills there was a significant decline of student performance in the subsequent clinical years, as they learn medical problem-solving $[18,19]$. This discrepancy is likely to be due to differences regarding courses; in our settings, knowledge on clinical sciences, clinical reasoning and problem-solving skills are learned concomitantly in clinical disciplines that use real clinical scenarios where the opportunities for practicing were numerous, which could not occur elsewhere.

Regarding the clinical skills related to interpretation of diagnostic tests, such as the electrocardiogram and chest radiographs, the significant decay herein observed is in accordance with the results for knowledge retention, since these clinical skills are essentially cognitive in nature. This finding is possibly also related to non use or non practice [1, 3-7], since the opportunities for practicing these skills in the semesters before the internship period are not so frequent. A monumental meta-analysis study focusing on the factors influencing the loss of trained skills or knowledge after prolonged periods of non use has found that after more than one year of non-practice, an individual might be performing at less than $92 \%$ of his performance level before the non-practice interval [6], a finding that applies to the our results.

As far as the physical examination skills are concerned, scores were the highest, since the baseline assessment (Table 2), thus indicating that the performance was always good. It is important to emphasize however that these scores derived from checklists items measuring only the exam technique and not detection and interpretation of the clinical signs found. Maintenance of these clinical skills is again most likely to be explained by continuous practice after the initial acquisition, since as, at least for skills related to the examination of the eye [21], additional training successfully reinforces performance, which progressively decays with non practice. Our findings, obtained in an educational scenario characterized by a richness of opportunities for practicing clinical skills, as already mentioned, are in accordance 
with those from a comparative study including students from four schools with different programs [32], which found that those from the one school with a longitudinal skills training program maintained higher scores in the subsequent clinical years. Again, the most likely interpretation for our findings showing maintenance or even improvement of genuine, but generic clinical skills is continued practice associated with student clinical work in the intervening period.

Taken as a whole, our findings are in accordance with the established notion that more physical and natural task-related skills, such as communication with patients and physical examination were less susceptible to loss than knowledge or cognitive abilities [6]. Assuming that non use is likely to be the cause for the knowledge decay, and student practice is the probable reason for maintenance of performance on clinical skills, the logical educational implication of our findings is in support of instructional strategies favoring both repetitive studying or refresher teaching and continuous practice. Also, repetitive testing could not only promote retention but also be instrumental in documenting knowledge and skills loss, which could therefore prompt opportune intervention.

Our study has a number of limitations that need to be taken into account. The sample size was relatively small and refers to a single class of a single medical school, which was studied over a relatively short period. Also, the accuracy of our clinical skills examination could also not be determined very precisely. Moreover, our findings come from a traditional medical school, so that these results cannot be generalised to educational scenarios characterised by the use of more active, student-centre learning strategies. Therefore, further studies are needed to confirm the results obtained and to ascertain whether they could be generalised to other educational settings. Also, the factors involved in decay or retention of knowledge and clinical skills, such as motivation and individual learner characteristics, teaching methods, learning environment, maintenance practice and retention interval, among others, should be specifically investigated in a more comprehensive manner.

\section{CONCLUSIONS}

Our data has showed that medical student retention of specific clinical sciences knowledge and cognitive skills related to diagnostic interpretation tests follow a pattern similar to that found in other scientific domains, characterized by progressive decay after initial acquisition. In contrast, fundamental clinical skills, such as communication with patients and physical examination seem to be substantially retained for at least one year, which could be ascribed to continuous practice. Assuming that these findings are causally related to use or non use of knowledge and skills rehearsal, respectively, the results of the present work supports educational strategies favoring both repetitive student re-exposure to previously learned contents and continuous practice.

\section{LIST OF ABBREVIATIONS}

$\begin{array}{lll}\text { MCQ } & =\text { multiple choice questions; } \\ \text { OSCE } & =\text { objective, structured clinical examination; } \\ \text { UNAERP } & =\text { University of Ribeirao Preto. }\end{array}$

HCRP $=$ Hospital das Clinicas de Ribeirao Preto (Ribeirao Preto University Hospital)

\section{COMPETING INTERESTS}

The authors declare that they have no competing interests.

\section{AUTHORS' CONTRIBUTIONS}

Both authors were responsible for the design of the study and the interpretation of data. FA made most of the data collection. LEAT performed the statistical analysis. Both authors worked together on successive versions up to the final manuscript

\section{ACKNOWLEDGEMENTS}

The authors would like to thank Mr Luiz Scarparo Neto, the student volunteers and staff members, together with the Phonoaudiology Department of the University of Ribeirao Preto (UNAERP) for their invaluable help.

\section{REFERENCES}

[1] Custers EJ. Long-term retention of basic science knowledge: a review study. Adv Health Sci Educ Theory Pract 2010; 15(1): 10928.

[2] Kennedy WB, Kelley PR, Saffran M. Use of NBME examinations to assess retention of basic science knowledge. J Med Educ 1981; 56(3): 167-73.

[3] Rico E, Galindo J, Marset P. Remembering biochemistry: a study of the patterns of loss of biochemical knowledge in medical students. Biochem Educ 1981; 9(3): 100-102.

[4] Custers EJFM, Ten Cate OTJ. Very long-term retention of basic science knowledge in doctors after graduation. Med Educ 2011: 45(4): 422-30.

[5] Friedlander MJ, Andrews L, Armstrong EG et al. What can medical education learn from the neurobiology of learning? Acad Med 2011; 86(4): 415-20.

[6] Arthur W, Bennett W, Stanush PL, McNelly TL. Factors that influence skill decay and retention: a quantitative review and analysis. Human Performance 1998; 11(1): 57-101.

[7] Hikosaka O, Rand MK, Nakamura K, et al. Long-term retention of motor skills in macaque monkeys and humans. Exp Brain Res 2002; 147(4): 494-504.

[8] Stross JK. Maintaining competency in advanced cardiac life support skills. JAMA 1983; 249(24): 3339-41.

[9] Wayne DB, Siddall VJ, Butter J, et al. A longitudinal study of internal medicine resident's retention of advanced cardiac life support skills. Acad Med 2006;81(10 Suppl): S9-S12.

[10] Blumenfeld A, Ben Abraham R, Stein M, et al. Cognitive knowledge decline after Advanced Trauma Life Support courses. J Trauma 1998; 44(3): 513-16.

[11] Kneebone R, ApSimon D. Surgical skills training: simulation and multimedia combined. Med Educ 2001; 35(9): 909-15.

[12] Stefanidis D, Korndorffer JR, Sierra R, Touchard C, Dunne JB, Scott DJ. Skill retention following proficiency-based laparoscopic simulator training. Surgery 2005; 138(2): 165-70.

[13] Wheeler DW, Degnan BA, Murray LJ et al. Retention of drug administration skills after intensive teaching. Anaesthesia 2008; 63(4): 379-84.

[14] Kromann CB, Bohnstedt C, Jensen ML, Ringsted C. The testing effect on skills learning might last 6 months. Adv Health Sci Educ Theory Pract 2010; 15: 395-401.

[15] Barsuk JH, Cohen ER, McGaghie WC, Wayne DB. Long-term retention of central venous catheter insertion skills after simulationbased mastery learning. Acad Med 2010; 85 (10) (Suppl 10): S9S12.

[16] Vadnais MA, Dodge LE, Awtrey CS, Ricciotti HA, Golen TH, Hacker MR. Assessment of long-term knowledge retention follow- 
ing single-day simulation training for uncommon but critical obstetrical events. J Matern Fetal Neonatal Med 2012; 25(9): 1640-5.

[17] Turner NM, Scheffer R, Custers E, Cate OT. Use of unannounced spaced telephone testing to improve retention of knowledge after life-support courses. Med Teach 2011; 33(9): 731-7.

[18] Engler CM, Saltzman GA, Walker ML, Wolf FM. Medical student acquisition and retention of communication and interviewing skills. J Med Educ 1981; 56(7): 572-9.

[19] Craig JL. Retention of interviewing skills learned by first-year medical students: a longitudinal study. Med Educ 1992; 26(4): 27681.

[20] Pfeiffer C, Madray H, Ardolino A, Willms J. The rise and fall of students' skill in obtaining a medical history. Med Educ 1998; 32(3): 283-8.

[21] Mottow-Lippa L, Boker JR, Stephens F. A prospective study of the longitudinal effects of an embedded specialty curriculum on physical examination skills using an ophthalmology model. Acad Med 2009; 84(11):1622-30.

[22] Haladyna TM, Downing SM, Rodriguez MC. A review of multiplechoice item-writing guidelines for classroom assessment. Appl Meas Educ 2002; 15(3): 309-34.

[23] Harden RM, Stevenson M, Downie WW, Wilson GM. Assessment of clinical competence using objective structured examination. $\mathrm{Br}$ Med J 1975; 1(5955): 447-51.
[24] Troncon LEA. Clinical skills assessment: limitations to the introduction of an "OSCE" (Objective Structured Clinical Examination) in a traditional Brazilian medical school. Sao Paulo Med J 2004; 122(1): 12-17.

[25] Barrows HC. Simulated (standardized) patients and other human simulations. Chapel Hill: Health Sciences Consortium 1987.

[26] Downing SM. Validity: on the meaningful interpretation of assessment data. Med Educ 2003; 37(9): 830-7.

[27] Downing SM. Reliability: on the reproducibility of assessment data. Med Educ 2004; 38(9):1006-12.

[28] Schmidmaier R, Ebersbach R, Schiller M, Hege I, Holzer M, Fischer MR. Using electronic flashcards to promote learning in medical students: retesting versus restudying. Med Educ 2011; 45(11): 1101-10.

[29] Perloff JK. The clinical recognition of congenital heart disease. Philadelphia: W. B. Saunders Co. 1970.

[30] Anderson J, Graham A. A problem in medical education: is there an information overload? Med Educ 1980; 14(1): 4-7.

[31] Ali J, Cohen R, Adam R et al. Attrition of cognitive and trauma management skills after the Advanced Trauma Life Support (ATLS) course. J Trauma 1996; 40(6): 860-6.

[32] Remmen R, Scherpbier A, van der Vleuten C et al. Effectiveness of basic clinical skills training programmes: a cross-sectional comparison of four medical schools. Med Educ 2001; 35(2): 121-8.

(c) Amaral and Troncon; Licensee Bentham Open.

This is an open access article licensed under the terms of the Creative Commons Attribution Non-Commercial License (http://creativecommons.org/licenses/by-nc/3.0/) which permits unrestricted, non-commercial use, distribution and reproduction in any medium, provided the work is properly cited. 